\title{
Achieving a Patient-Centered, Value-Based System of Diabetes Care in the United States: Report on a National Stakeholder Summit
}

\author{
David B. Nash, MD, MBA, Alexis Skoufalos, EdD, and Dennis Harris, PhD²
}

\begin{abstract}
Chronic instability in the health care environment has raised concerns among providers and patients, especially those who treat and cope with chronic conditions. Changes to existing health care laws are unlikely to alter what have become the primary goals of the US health care system: cost-effectiveness and patient-centricity. To that end, it is vital that patient and physician voices be incorporated in policy decisions and, importantly, that access to care and patient-reported outcomes are considered when calculating "value." Following a discussion of perceived pressures on patient access to information and appropriate treatment for diabetes, a panel of engaged stakeholders in the diabetes community outlined and committed to a collaborative effort aimed at effecting necessary policy changes and ensuring that a patient-centered, value-based system of diabetes care is achieved. The overarching themes that emerged included: (1) patients and physicians must have a stronger voice and a place at the table; (2) a collaborative of multiple organizations is necessary to seize improvement opportunities; and (3) the diabetes community must advocate for population health initiatives around diabetes.
\end{abstract}

Keywords: policy, patient-centered, value-based, diabetes

\section{Introduction}

$\mathbf{T}$ HE CHALLENGE OF managing chronic disease is unrelenting and most changes to health care, regardless of predictability, are problematic. The recent political and policy-driven upheaval within the health care environment has made it particularly difficult for patients and providers to ensure uninterrupted and uncompromised care. The challenges are intensified for populations with chronic illnesses, who typically grapple with complex ongoing medical and behavioral health needs. Characterized by the Centers for Disease Control and Prevention (CDC) as a US epidemic, diabetes mellitus is a prime example of a chronic condition with broad ranging population health and economic implications.

Statistics show that in 2016 more than 29 million Americans $(9.3 \%$ of the population) were living with type 1 (approximately $5 \%$ ) or type 2 (approximately 95\%) diabetes. ${ }^{1}$ The health and economic costs of both types are enormous. Diabetes is the seventh leading cause of death and the leading cause of kidney failure, lower-limb amputations and adult-onset blindness. Other complications associated with diabetes include heart disease and stroke. ${ }^{2}$ In economic terms, the total cost of diagnosed diabetes in the United States is $\$ 245$ billion; $\$ 176$ billion in direct medical costs and $\$ 69$ billion in reduced productivity. Age- and genderadjusted average medical expenditures among people with diabetes are 2.3 times higher than expenditures would be in the absence of diabetes. ${ }^{1,2}$

Substantial effort and coordination is required on the part of both the patient and the provider to manage diabetes effectively. In addition to making multiple daily decisions regarding food intake, physical activity, and medications, a person with diabetes must be proficient in a variety of selfmanagement skills (eg, monitoring blood glucose and predicting changes, understanding and calculating carbohydrate content, recognizing signs of hypo- and hyperglycemia). Because many patients with diabetes have comorbidities, clinicians are faced with complex medical management decisions (eg, tailoring therapy to minimize intolerances and adverse effects, managing polypharmacy, adjusting myriad

\footnotetext{
${ }^{1}$ Jefferson College of Population Health, Philadelphia, Pennsylvania.

${ }^{2}$ The Endocrine Society, Washington, District of Columbia.
}

(C) David B. Nash et al. 2018; Published by Mary Ann Liebert, Inc. This is an Open Access article distributed under the terms of the Creative Commons Attribution License, which permits unrestricted use, distribution, and reproduction in any medium, provided the original work is properly cited. 
medications in the face of complications as the disease progresses). ${ }^{3}$ It is vital that clinicians and patients understand the complexity of diabetes and work together to determine the most effective treatment for each individual.

All efforts toward achieving a more patient-centered, value-based system of diabetes care assume sufficient access to care. Access to health care implies having "the timely use of personal health services to achieve the best health outcomes." 4 Attaining appropriate access to care requires 3 distinct steps: (1) gaining entry into the health care system; (2) getting access to care settings where the patient can receive needed services; and (3) finding providers who meet the needs of individual patients and with whom patients can develop a relationship based on mutual communication and trust. ${ }^{5}$

\section{Listening to the Voices of Patients and Their Providers}

Quality, cost-effectiveness and patient-centricity are likely to remain the primary goals of the health care system, regardless of changes to federal health care legislation. A successful transition to a more patient-centered, value-based system of diabetes care requires that all stakeholders - and the diabetes advocacy community in particular - work collaboratively to assure that patients have affordable access to products and services that treat and help them effectively manage diabetes to prevent or delay the development of serious complications.

Ensuring that patients have access to care that their physicians deem necessary is a foundational pillar of the medical management of diabetes. To that end, current disparate efforts among stakeholder groups must become better aligned and focused so that models of care, policies, pricing and reimbursement structures promote and encourage innovation. Emphasis must be placed on promoting optimal treatment strategies, therapies and services that provide the best outcomes for individual patients based on objective measures. As in other priority areas, rapid innovation and discovery of medical management models, health technology and pharmaceuticals must be encouraged with an eye toward maximum health benefit for persons with diabetes.

Building upon an ongoing series of discussions and expert panel meetings, the Jefferson College of Population Health (JCPH) convened a meeting of representatives from diabetes advocacy groups, professional societies, and population health and health policy experts to explore how all parties might work collaboratively to define the issues and develop more efficient and effective approaches to creating and delivering information to patients to help them access appropriate diabetes care.

Process experts included: David B. Nash, MD, MBA (Dean, JCPH), Facilitator; Stephen Teller, MBA (Parallax Life Sciences Consulting, LLC); and Alexis Skoufalos, EdD (Associate Dean, JCPH). Participating stakeholders included:

AMGA - represented by Jerry Penso, MD, MBA, Chief Medical and Quality Officer

American Pharmacists Association Foundation (APhAF) - represented by Ben Bluml, RPh, Sr. Vice President, Research and Innovation

Children with Diabetes Association - represented by Jeff Hitchcock, BS, President

Close Concerns/The diaTribe Foundation - represented by Sarah Odeh, BS, Vice President for Operations
College Diabetes Network - represented by Christina Roth, CEO and Founder

Diabetes Sisters - represented by Anna Norton, MS, CEO

D-Pac - represented by Bennett Dunlap, MS, Patient Advocate

Endocrine Society - represented by Dennis Harris, PhD, Associate Director, Content Strategy

Juvenile Diabetes Research Foundation - represented by Jessica Roth, MPH, Senior Director, Health Policy

Pharmacy Benefit Management - represented by Dea Belazi, PharmD, MPH, CEO, AscellaHealth

Sustainable Healthy Communities, LLC - Laura Lee Hall, $\mathrm{PhD}, \mathrm{COO}$

First Samurai Consulting, LLC - Kenneth Moritsugu, MD, MPH, FACPM, President and CEO

T1D Exchange - represented by Jill Petrie, Director of Communications and Community Relations

\section{Perceived Pressures on Patient Access}

A variety of issues fomented by the unpredictable health care climate may impede patient access to appropriate diabetes care. These include a serious gap in education and awareness among providers and their patients with diabetes, a lack of transparency regarding pricing and formulary decisions, cumulative increases in health insurance premiums relative to income, increased cost-shifting to beneficiaries and the potential for restricted access to new or current medicines. The "safety net" population is especially vulnerable and at the greatest risk in these changing times. Patients living in economically challenged areas and those who are less able to care for themselves must be considered by public and private policy makers.

A pre-meeting survey, administered to participants the month prior to the meeting, was designed to help focus the agenda on areas of primary interest to the group. All respondents cited rising drug prices and pressure on access to diabetes medications as key market/policy challenges. Other important issues included lack of patient input regarding value models used for payer decision making, and the impact of follow-on biologics and medication switching.

\section{Follow-on biologics and individual patient variability}

The still evolving regulatory processes and directions concerning biological and follow-on biological drugs has created general confusion among providers and patients with diabetes. Of key concern was the anticipated change in the US Food and Drug Administration's process for evaluating and approving follow-on biologics and its potential effect on patient access to appropriate therapies. Specifically, there are complexities associated with transitioning from the current evaluation process to an approval process whereby biologic medicines seeking biosimilar status (including insulins) will be routed through an evolving biologic pathway beginning in 2020. The law does not take patient outcomes into account and its application is likely to vary by state.

Individuals with diabetes have unique responses to various insulins and often spend considerable time working with their health care providers to determine which insulin preparation is most appropriate and effective for them. A premeeting survey of expert panelists revealed concerns about the potential for large insurers to exclude currently available 
insulin products from formularies primarily on the basis of price without considering variability of individual patient response. Meeting participants reasoned that the prospect of insurer and pharmacy benefit manager reliance on nonmedical rationales to incent medication switching would have a negative impact on patient health outcomes, and the patientphysician decision-making process would be disrupted. The primary safety concern of patient advocate groups is that antigenicity, or immune response, for any one patient, will differ unpredictably for different follow-on biologics for the same reference drug. ${ }^{6}$

These issues have arisen in connection with human growth hormone $(\mathrm{hGH})$, a biologic drug produced by 10 different manufacturers. Studies show that despite the availability of clinical guidelines for the use of $\mathrm{hGH}$, there is a gap between providers' recommendations and their patients' access to prescribed treatment due, in part, to the cost of a specific drug and insurers' desire to limit its use. ${ }^{7,8}$

The Biologics Prescribers Collaborative has made a positive impact on policy on access to biologics, including follow-on biologics and biosimilars, and nonmedical medication switching in the treatment of chronic diseases. The Endocrine Society, one of 6 member organizations in the collaborative, might be helpful in making the case in support of policies that support the safest possible use of biologics in the treatment of diabetes (more information available at http://biologicsprescribers.org).

\section{Importance of Collaboration}

The collective survey response was unanimous regarding the chief barrier hampering advocacy - fragmentation of the advocacy community and difficulty in coordinating efforts. Many advocates do not fully understand the complex drivers and issues beyond their specific vantage points and, without a unified "voice" or spokespersons able to articulate patient and provider concerns, advocacy stakeholders have found it difficult to develop comprehensive resources, capture the attention of payers and impact policy in a meaningful way. The work of the advocacy community is further complicated by the underlying problem of fragmentation in the health care delivery system.

The pre-meeting survey revealed opportunities for improving communication and collaboration among diabetes patient advocacy organizations. For example, a majority of survey respondents were unaware of multiple resources and initiatives that are available, under way or planned. These include patient self-advocacy resources (eg, diaTribe's payer playbook tool, Diabetes Sisters' patient navigation tool kit, Friends for Life Conferences) and payer accountability initiatives (eg, publicly available report cards, HEDIS measures, CMS Star ratings, JDRF policy resources focused on payer initiatives, employer/broker spreadsheets that detail the cost of therapies).

Beyond facts and figures, meeting participants concurred that successful disease state change requires a strong, collective voice and a "face" to interact effectively with policy decision makers and politicians. Patients facing the financial challenges of managing their diabetes and accessing care must become involved in the policy-making process. The advocacy community can support patients by helping them to become better informed consumers who are capable of persuading policy makers to make meaningful improvements.

Recognizing the importance of collaboration among stakeholders on improving policies that affect patient access to care and resources to amplify a common message, the meeting participants drew upon the experience of advocates in other therapeutic areas in recommending the following actions: (1) identify voids in the advocacy effort; (2) determine 1-2 initiatives that a variety of advocates can support; (3) establish a collaborative structure for combining and applying resources; and (4) set interim goals for rapid "wins" and maintaining momentum.

\section{Common Goals and Action Plans}

Participants agreed to focus on 2 priority areas:

1) Creating resources to help patients advocate for themselves when facing changes in health insurance coverage.

Patient and physician resources and tools (eg, checklists, cost calculators, playbooks, success stories, score cards, "how-to" instructions) are important for ensuring access to necessary care. The primary initiative is to move from an unrelated set of disparate tools issued by many organizations to a collaboratively created, endorsed, branded, distributable product that is housed centrally and accessible to all organizations. Recognizing the ineffectiveness of "one-size-fitsall" tool kits, the initiative will address access challenges including vulnerable populations (eg, patients living in remote rural areas, patients living in poverty, patients requiring intensive diabetes therapies or approaches to care) and barriers to patient activation (differences in demographic characteristics, health literacy, and culture).

2) Developing a strategy to assess and hold payers (health plans, employers, and others) accountable for ensuring patient access to individualized, evidence-based care.

To improve accountability of payers to patients, the collective patient voice must be amplified and a public conversation must begin. "Crowdsourced" data from the diabetes advocacy community can be collected to help payers better understand the impact and cost consequences of medication changes on patients. Such aggregated data collection and analysis can have a powerful impact in terms of promoting transparency, bringing holistic cost information to payers, promoting public conversation and incorporating the patient experience in payment decisions. The feasibility of a common collaborative platform will be investigated to help pool resources and facilitate advocacy efforts toward improving access to diabetes therapies.

\section{Conclusion}

Across the United States, persons with diabetes and their providers face multiple challenges in accessing necessary care. For these stakeholders, this includes easy access to patient-centric preventive services, education, and therapies. The unpredictable health care environment has created a sense of urgency among providers and patients dealing with chronic conditions. Regardless of changes to existing health care laws, cost-effectiveness and patient-centricity are likely to remain the primary goals of the health care system. It is vital that patient and physician voices be incorporated in 
policy decisions and that patient-reported outcomes are considered when calculating "value." A panel of experts representing diabetes advocacy groups, professional societies, and population health and health policy teams agreed that there are substantial pressures on patient access to information and appropriate treatment. They mapped out a concerted, coordinated effort aimed at effecting necessary policy changes and ensuring that a patient-centered, value-based system of diabetes care is achieved. Three important themes emerged over the course of the meeting: (1) patients and physicians must have a stronger voice and a place at the table; (2) a collaborative of multiple organizations is necessary to seize improvement opportunities; and (3) the diabetes community must advocate for population health initiatives around diabetes.

\section{Author Disclosure Statement}

The authors declare the following potential conflicts of interest: Drs Nash and Skoufalos are employed by the Jefferson College of Population Health (JCPH). Jefferson College of Population Health received financial support from Sanofi to convene and organize the expert panel meeting and to develop this manuscript. Dr Harris was a participant at the expert panel meeting. Expert panelists did not receive compensation for participation in the meeting. Sanofi reimbursed expert panelists for their travel expenses.

\section{References}

1. Centers for Disease Control and Prevention. Diabetes. Working to reverse the US epidemic - at a glance 2016. www.cdc.gov/chronicdisease/resources/publications/aag/pdf/ 2016/diabetes-aag.pdf Accessed May 1, 2017.
2. American Diabetes Association. Statistics about diabetes. www.diabetes.org/diabetes-basics/statistics Accessed May 1, 2017.

3. Kennedy M, Roberts A. Complex type 2 diabetes mellitusmanagement challenges and pitfalls. Aust Fam Physician 2013;42:207-210.

4. Institute of Medicine; Committee on Monitoring Access to Personal Health Care Services. Access to Health Care in America. Washington, DC: National Academies Press; 1993.

5. Agency for Healthcare Research and Quality. 2011 National Healthcare Quality Report. Chapter 9: Access to care. October 2014. www.ahrq.gov/research/findings/nhqrdr/nhqr11/ chap9.html Accessed June 5, 2017.

6. Basskin L. Biosimilars-substitution is not just for your grandfather's generics anymore. www.pharmacytimes.com/ publications/directions-in-pharmacy/2015/october2015/ biosimilarssubstitution-is-not-just-for-your-grandfathersgenerics-anymore Accessed June 12, 2017.

7. Chidambaram A. Product differentiation to play a vital role in Europe. May/June 2013. www.researchgate.net/publication/ 304092504_Human_Growth_Hormones_Product_Differentiation_ to_Play_a_Vital_Role_in_Europe Accessed June 12, 2017.

8. Rose SR, Cook DM, Fine, MJ. Growth hormone therapy guidelines: clinical and managed care perspectives. October 24, 2014. www.ajpb.com/printer?url=/journals/ajpb/2014/ ajpb_septemberoctober2014/growth-hormone-therapyguidelines-clinical-and-managed-care-perspectives Accessed June 12, 2017.

Address correspondence to: Alexis Skoufalos, EdD Jefferson College of Population Health 901 Walnut Street, 10th Floor Philadelphia, PA 19107

E-mail: alexis.skoufalos@jefferson.edu 